\title{
Editorial
}

\section{Full-Duplex and Cognitive Radio Networking for the Emerging 5G Systems}

\author{
M. Shikh-Bahaei $\mathbb{D}^{1},{ }^{1}$ Y.-S. Choi ${ }^{D},{ }^{2}$ and D. Hong ${ }^{3}$ \\ ${ }^{1}$ Department of Informatics, King's College London, 30 Aldwych, London WC2B 4BG, UK \\ ${ }^{2}$ Intel Labs, Hillsboro, OR, USA \\ ${ }^{3}$ Department of Electrical and Electronic Engineering, Yonsei University, 134 Sinchon-dong, Seodaemun-gu, \\ Seoul 120-749, Republic of Korea \\ Correspondence should be addressed to M. Shikh-Bahaei; m.sbahaei@kcl.ac.uk
}

Received 4 February 2018; Accepted 6 February 2018; Published 4 March 2018

Copyright (c) 2018 M. Shikh-Bahaei et al. This is an open access article distributed under the Creative Commons Attribution License, which permits unrestricted use, distribution, and reproduction in any medium, provided the original work is properly cited.

Full duplex and cognitive radio technologies have paved the way to enormous advances in wireless communications. Cognitive radio, since 1990s, has predicted transceivers that can be aware of their surroundings and be able to detect and exploit underutilized frequency bands licensed to other users. With half duplex radios, sensing the primary channels, detecting spectrum holes, and accessing the idle or underutilized channels according to certain algorithms are performed sequentially by cognitive (secondary) users. This can inherently cause interfering with primary users in the licensed bands, as secondary users cannot hear the return of primary users while transmitting data.

With full duplex radio, on the other hand, the users can transmit data and sense/receive data simultaneously. This can potentially improve the throughput of the secondary network and at the same time provide better protection to the primary users. Since the secondary users can sense the primary channel(s) whilst transmitting over the primary's idle channel, they can detect return of primary users and would immediately stop transmission over the respective frequency band. End to end delay reduction is also another major benefit of full duplex communication, as some of the above sequential processes can be made concurrent with full duplex radio.

Potential benefits of full duplex communication, specifically in enhancing spectral efficiency, have also been known since 1960s. However, hardware and system complexities hindered wide use of these technologies in realistic conditions; substantial effect of self-interference in full duplex radio and practical difficulties in cancelling it were the prime reasons for the five-decade delay in serious attention to this technology. Recent advances in self-interference cancellation, through cutting edge digital and analog signal processing techniques, have brought full duplex communication closer to reality. At the same time, remarkable advances in centralized and distributed signal processing, machine to machine communication, deep learning methods, and cloud/edge computing have spurred a major shift in cognitive radio paradigm and realized many early promises of this technology.

Applications of these two technologies, in the future wireless networks, can be vast, and the potentials for improving network performance, for example, in terms of delay and throughput, are promising. As said above, by combining the two technologies, that is, cognitive and full duplex radio, even further benefits can be achieved, for instance, offering higher secondary user throughput and concurrently protecting the primary users through parallel sensing and transmitting data by the secondary users.

However, when we deal with 4 th and 5 th generation dense networks and apply full duplex and cognitive radio technologies in the emerging Internet of Things, new challenges will arise. For instance, multiuser interference is increased when full duplex communication is exploited in multiuser scenarios, and especially in dense environments. This will necessitate new cognitive and full duplex MACand Network-layer protocols to tackle these problems in multiuser and multihop scenarios. Such new MAC and Network layer protocols can be far more efficient than the respective standard protocols, as the users can learn the 
environment through cognitive radio and can transmit while sensing through full duplex radio.

Security is another concern in wireless networks with potentially large number of users/machines competing for limited shared pool of resources, specifically in shared spectrum cognitive network conditions.

The present special issue of this journal is dedicated to addressing some of the challenges in cognitive and full duplex networking. The first paper "Cognitive Security of Wireless Communication Systems in the Physical Layer" by M. H. Yilmaz et. al. looks into so-called "cognitive security" and proposes new physical layer measures for enhancing security in cognitive wireless networks. This paper specifically suggests employing an adaptive radio that adapts to the network condition and accordingly takes necessary precautions before security attack takes place.

The second paper, "Fast Cooperative Energy Detection under Accuracy Constraints in Cognitive Radio Networks" by $\mathrm{S}$. Peng et al., studies minimizing the delay incurred in finding and accessing frequency holes in cognitive networks. Service latency is a critical constraint in many of the applications in the 5th generation wireless systems. The proposed technique in this work is based on cooperative energy detection by the secondary (i.e., cognitive) users.

The third paper, "Coexistence of Cognitive Small Cell and WiFi System: A Traffic Balancing Dual-Access Resource Allocation Scheme" by X. Huang et al., touches upon optimization of heterogeneous cognitive networks, comprising (licensed) small cells and (unlicensed) wifi constituents. 5G networks are envisaged to corroborate the idea of cognitive networking in heterogeneous conditions; hence optimal allocation of resources in such networks is essential for achieving fair and high quality of service provisioning to various applications. New power allocation algorithms are presented by the authors to achieve traffic balance between licensed and unlicensed users in a cognitive networking setup.

The 4th paper, "Distributed Schemes for Crowdsourcingbased Sensing Task Assignment in Cognitive Radio Networks" by L. Zhai et al. studies crowdsourcing by exploiting spectrum sensing in cognitive wireless networks. This paper considers the scenario where spectrum sensing is assigned to mobile terminals and tablets as a distributed task. The authors propose a number of algorithms in order to optimize this assignment.

The last paper, "Cooperative Full-Duplex Physical and MAC Layer Design in Asynchronous Cognitive Networks" by $\mathrm{T}$. Febrianto et. al., studies an asynchronous cognitive network (i.e., no synchronicity between primary and secondary users), where the nodes are also capable of full duplex communication. Full duplex radio in cognitive users has been exploited for enhancing the sum-throughput of the network. The users can simultaneously transmit and sense channel/receive data. This paper also proposes a new full duplex MAC protocol for the case where multiple secondary users cooperatively sense the primary channels. This paper considers the case where a cognitive full duplex user can sense multiple channels and studies the effect of the number of sensed channels on the MAC throughput.

M. Shikh-Bahaei

Y.-S. Choi

D. Hong 


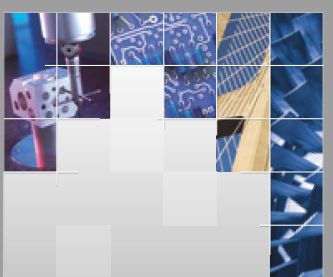

\section{Enfincering}
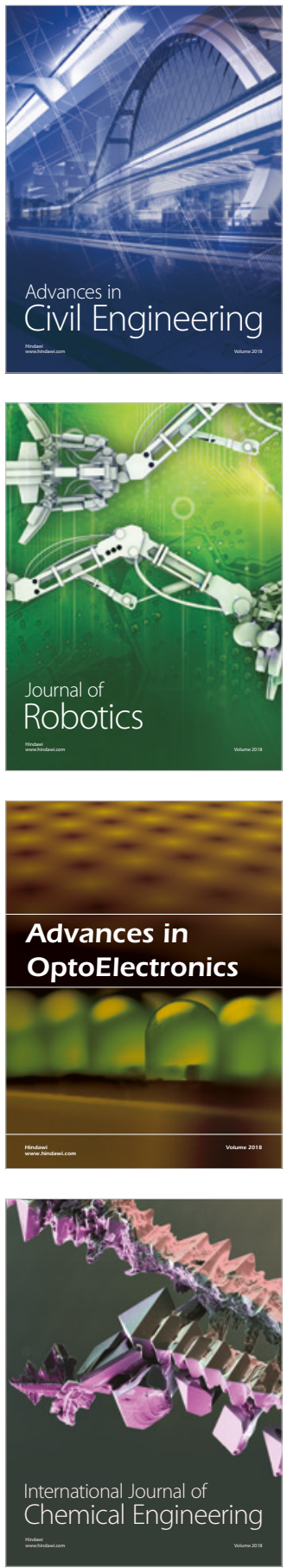

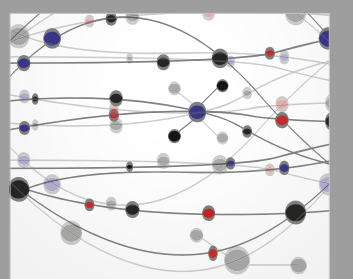

\section{Rotating \\ Machinery}

The Scientific World Journal

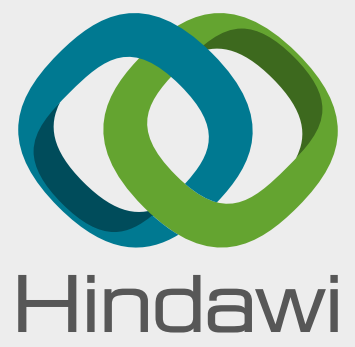

Submit your manuscripts at

www.hindawi.com
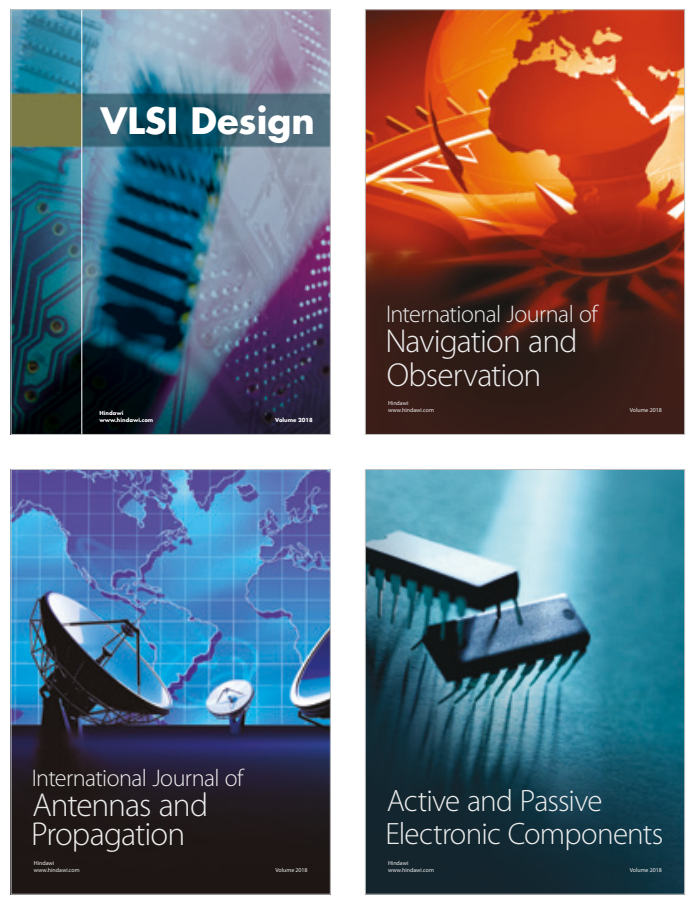
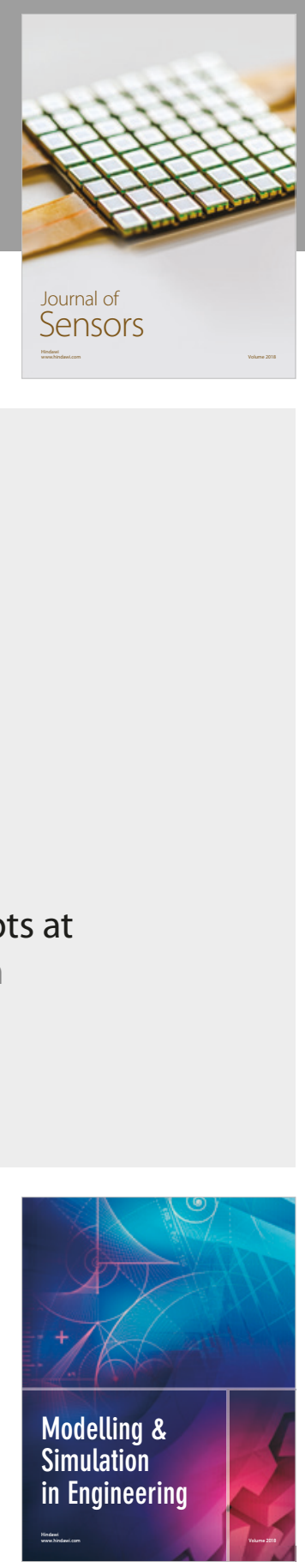

\section{Advances \\ Multimedia}
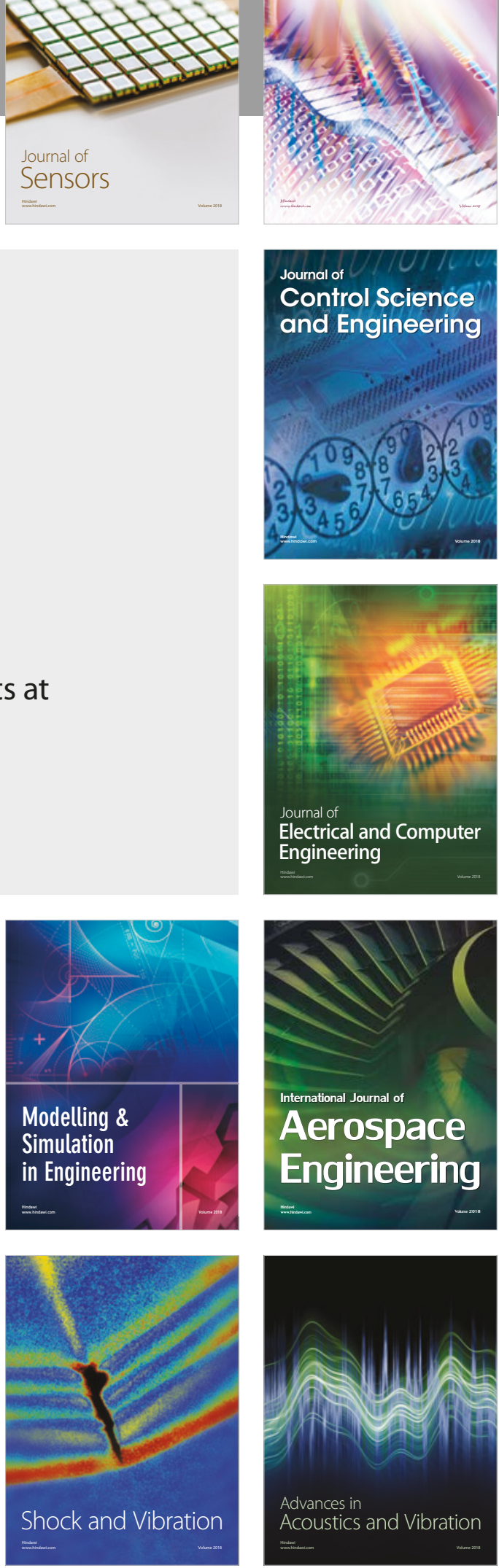